\title{
Lidil
}

Revue de linguistique et de didactique des langues

$45 \mid 2012$

Pratiques de formation à la lecture-écriture des adultes en parcours d'insertion

\section{Illettrisme, évaluation et formation : réflexions sur une approche européenne}

Marie-Hélène Luis, Sara Majaji, Cynthia Ciappara-Diaz et Jean-Marie Besse

\section{CpenEdition}

\section{Journals}

Édition électronique

URL : http://journals.openedition.org/lidil/3171

DOI : 10.4000/lidil.3171

ISSN : 1960-6052

Éditeur

UGA Éditions/Université Grenoble Alpes

Édition imprimée

Date de publication : 15 mai 2012

Pagination : 13-25

ISBN : 978-2-84310-226-4

ISSN : $1146-6480$

Référence électronique

Marie-Hélène Luis, Sara Majaji, Cynthia Ciappara-Diaz et Jean-Marie Besse, « Illettrisme, évaluation et formation : réflexions sur une approche européenne », Lidil [En ligne], 45 | 2012, mis en ligne le 15 novembre 2013, consulté le 19 avril 2019. URL : http://journals.openedition.org/lidil/3171 ; DOI : 10.4000/lidil.3171 


\title{
Illettrisme, évaluation et formation : réflexions sur une approche européenne
}

\author{
Marie-Hélène Luis, Sara Majaji, \\ Cynthia Ciappara-Diaz et Jean-Marie Besse*
}

\begin{abstract}
Résumé
Le PsyEF, équipe de recherche à l'université Lyon 2, a participé à trois projets européens de formation d'adultes. Après la construction des projets et leur intérêt pour l'équipe, le présent article détaille le partenariat et aborde les contraintes d'une recherche conduite dans des pays aux cultures et aux langues différentes. Sont exposés également les avantages d'une dynamique de coopération, les adaptations nécessaires et les exigences de la diffusion sur internet. Compte tenu de l'évolution et de l'internationalisation des pratiques de formation, il semble utile de maintenir des recherches au niveau européen; à cet égard, les travaux des trois projets représentent une avancée pour la réflexion sur les compétences des personnes en difficulté et sur les profils de formateurs.
\end{abstract}

\section{ABSTRACT}

The PsyEF, a research team at the University Lyon 2, took part in three European projects for adult education. After detailing the development of the projects and their interest for the team, this article describes the partnership and discusses the difficulties of research conducted in countries with different cultures and languages. The benefits of a dynamic cooperation, the necessary adjustments and the requirements of the webcasting are explained. Given the evolution and internationalization of practices in training programs, it appears helpful to maintain researches at European level and in this respect, the work of the three projects represents a headway for the thought about the skills of people with learning difficulties and the profiles of trainers.

* Membres du PsyEF, Université Lumière-Lyon 2 : Marie-Hélène Luis, docteur en sciences du langage ; Sara Majaji, psychologue, doctorante en psychologie ; Cynthia Diaz-Ciappara, psychologue ; Jean-Marie-Besse, fondateur et directeur du PsyEF, professeur de psychologie. 
La stratégie de Lisbonne ${ }^{1}$ pour la modernisation du modèle socioéconomique européen vise la constitution d'une « société de la connaissance ». C'est dans ce contexte que se sont construits des projets européens de formation. La commission européenne propose en effet des programmes pour l'éducation des adultes et finance des activités et des recherches afin, entre autres, d'améliorer l'offre de formation tout au long de la vie. Notre équipe française, le PsyEF ${ }^{2}$, a participé, de 2005 à 2010, à trois projets européens visant à coordonner des expériences et à proposer des perspectives dans ces domaines : le projet CEPPAC $3^{3}$ et les deux projets MODEVAL1 et MODEVAL2 ${ }^{4}$. Cet article a pour but d'analyser la dynamique de coopération des équipes appelées à travailler au sein de ces projets, l'intérêt et les limites de ces travaux ainsi que les perspectives envisagées à moyen terme par notre équipe.

\section{Construction des projets}

Le recrutement des équipes, pour les trois projets auxquels le PsyEF a participé, a été effectué par un coordinateur de projet appartenant à l'organisme AGORA ${ }^{5}$ et par un représentant de l'ANLCI $^{6}$. Ils ont approché des partenaires qui avaient déjà travaillé sur d'autres projets, des chercheurs ou praticiens qui leur avaient été recommandés, des intervenants à des colloques sur les compétences de base ou des auteurs d'ouvrages scientifiques reconnus par les communautés universitaires de divers pays. Les équipes européennes pressenties devaient être concernées par les adultes en difficulté sur l'écrit mais elles étaient également validées sur des critères linguistiques (les membres devaient pratiquer le français ou l'anglais quelle que soit la langue dans laquelle ils exerçaient), institutionnels (les organismes devaient être officiellement reconnus),

1. Stratégie de Lisbonne et formation : Journal officiel de l'Union européenne 28.5.2009 C 119/2.

2. Le PsyEF est une équipe de recherche sur les questions d'éducation et de formation, rattachée au Laboratoire Santé, Individu, Société (SIS, E.A.M. 4128) - Université de Lyon.

3. CEPPAC : Coopération Européenne sur les Pratiques Pédagogiques pour Améliorer les Compétences des acteurs de formation continue et initiale.

4. MODEVAL : MODèle européen d'EVALuation des compétences de base.

5. AGORA : Agora est une entreprise de l'économie sociale implantée à Lorient, dans le Morbihan.

6. ANLCI : Agence Nationale de Lutte Contre l'Illettrisme. 
professionnels (il fallait disposer d'un public concerné par l'illettrisme), relationnels (il s'agissait d'échanger avec des personnes exerçant une profession différente, dans des cadres différents, etc.). Approché durant l'année 2004, le PsyEF a été intéressé par le projet MODEVAL1, et a posé sa candidature. Dans la partie du formulaire de candidature consacrée aux compétences spécifiques du partenaire candidat, le PsyEF a fait ressortir ses recherches et recherches-actions les plus saillantes concernant l'évaluation des personnes en difficultés sur l'écrit?

\section{Intérêt des projets pour le PsyEF}

Membres du PsyEF, à l'université Lumière-Lyon 2, nous travaillons sur l'appropriation de l'écrit et l'illettrisme depuis le début des années quatre-vingt. Les projets proposés par Agora et l'ANLCI, qui visaient à étudier ces questions selon une perspective européenne, nous offraient non seulement une possibilité de confronter notre approche théorique et nos pratiques à celles d'autres pays européens, mais aussi l'occasion d'approfondir notre réflexion grâce à de nouveaux défis. En effet, la pluralité des partenaires pressentis nous laissait entrevoir des questionnements sociaux, pédagogiques et linguistiques bénéfiques à l'évolution de nos travaux.

Dans les projets MODEVAL1 et MODEVAL2, il s'agissait d'élaborer un outil d'évaluation de l'illettrisme commun à plusieurs pays d'Europe (MODEVAL1), puis de former les formateurs appelés à intervenir auprès de publics en difficulté sur la littératie, notamment à utiliser cet outil d'évaluation (MODEVAL2). Le projet CEPPAC3, quant à lui, était destiné à construire un outil de formation de formateurs à partir des résultats d'un projet précédent, CEPPAC2, qui avait vu la réalisation d'une série de matériels destinés à des personnes adultes en difficulté sur l'écrit. Le cahier des charges stipulait la composition d'un manuel reprenant et uniformisant les travaux CEPPAC2 ainsi que la construction d'une formation de formateurs s'appuyant sur ce manuel.

7. Création, pour le ministère de la Justice, des épreuves d'évaluation d'une population de personnes incarcérées; formation à la passation des épreuves des enseignants intervenant dans les prisons concernées (1999). Création, pour l'Insee, d'une partie des épreuves de l'enquête IVQ - Information Vie Quotidienne - (en 2004). Publication d'un outil d'évaluation : Diagnostic des Modes d'Appropriation de l'écrit, guide pratique, Paris, Retz (2004). 
Pour CEPPAC3, le Psy $E F$, qui n'avait pas pris part à CEPPAC2, avait été sollicité en tant qu'expert de l'illettrisme, afin de superviser autant la réalisation du manuel que la formation de formateurs.

Une fois le projet accepté par la commission européenne à la suite d'un rapport rédigé par deux experts indépendants, le coordinateur rassemblait les participants au cours d'une réunion dite de démarrage : les partenaires devaient se rencontrer pour se connaitre et travailler ensemble. Durant deux ans, c'est dans une alternance de regroupements réguliers et d'échanges sur le réseau, essentiellement via des courriels, mais aussi sur une plateforme internet, que le travail a progressé. Sur la durée des trois projets - MODEVAL1 (2005-2006), CEPPAC3 (20072008), MODEVAL2 (2009-2010) - c'est-à-dire 5 ans, la méthode a évolué. En 2005, certains partenaires étaient peu habitués à échanger par internet : leur institution était peu ou mal équipée, leur pratique était éloignée de ce média. Ces problèmes avaient presque disparu à la fin du dernier projet, tant l'Internet en 2010 fait partie du quotidien. Toutefois force est de constater que les regroupements physiques ont toujours fait beaucoup avancer la réflexion. L'expérience de la visioconférence, qui pourrait constituer une alternative aux déplacements, n'a pas pu avoir lieu faute d'équipement adéquat.

\section{Le partenariat européen}

Le projet CEPPAC a rassemblé des équipes belge, danoise, espagnole, françaises (trois), hongroise, polonaise, suisse. Les deux projets MODEVAL ont mobilisé des équipes allemande, belge, danoise, espagnole, françaises (trois), grecque, hongroise, maltaise et polonaise. Comme on peut le constater sur le tableau en annexe, les trois projets ont réuni des partenaires communs ou différents. Ainsi, n'ont pas participé à CEPPAC3 les partenaires allemands, grecs et maltais ; n'ont pas participé à MODEVAL2 les partenaires polonais et belges, à MODEVAL (1 et 2) les partenaires suisses. En ce qui concerne la Suisse, d'ailleurs, qui ne fait pas partie de la communauté européenne, ses représentants étaient qualifiés de partenaires « silencieux »; leur participation n'était pas financée par la communauté européenne et ils devaient remplir des objectifs spécifiques déterminés par leur institution. D'autre part, l'équipe belge de MODEVAL1 était différente de celle de CEPPAC3, et l'équipe espagnole de MODEVAL2 était composée d'autres membres que ceux de MODEVAL1 et CEPPAC3. Au total le partenariat rassemblait dix pays, dix langues différentes parlées par les 
populations concernées, six universités, quatre instituts supérieurs de formation, deux centres associatifs de formation, deux agences d'organisation. Les langues officielles utilisées lors des échanges étaient l'anglais et le français.

La lecture du tableau montre que les trois projets rassemblaient des universités et des centres de formation. Les participants étaient chercheurs universitaires ou praticiens (directeurs d'organismes de formation, formateurs, psychologues) et représentaient des disciplines diverses toutes concernées par l'illettrisme : l'andragogie, la linguistique, l'orthophonie, la pédagogie, la psychologie, la sociologie. Les concepts et les méthodes ne différaient donc pas seulement d'un pays à l'autre mais aussi d'une discipline à l'autre.

Notre équipe, dans ses travaux, a toujours recherché l'interdisciplinarité. Dès sa création, elle a associé à la psychologie la linguistique et la sociologie. Toutefois il faut bien reconnaitre que l'interdisciplinarité est complexe à mettre en place dans l'université française. Nous avons donc vu, dans ces trois projets européens, la possibilité d'approfondir notre champ de recherche grâce à l'ouverture à des équipes de disciplines et de pays différents. Nous savions, évidemment, qu'un certain nombre d'obstacles se présenteraient au cours d'un travail de deux ans avec des équipes éloignées géographiquement, linguistiquement et culturellement les unes des autres.

\section{Des difficultés issues de la diversité}

Dans ce type de coopération, la communication exolingue constitue la première difficulté. Les populations concernées par les projets parlaient dix langues différentes. Si les partenaires français, belges et suisses s'exprimaient dans la langue de leur objet de recherche, les partenaires francophones ou anglophones du Danemark, d'Espagne, de Grèce, de Hongrie, de Malte, de Pologne, s'exprimaient dans une langue étrangère à leur objet. Or les participants concernés n'étaient pas tous linguistes et le matériel linguistique utilisé en français ou en anglais leur était souvent étranger dans leur propre langue. De plus, les deux langues du projet, l'anglais et le français, étaient diversement dominées par les participants. Au cours des réunions, les échanges étaient traduits en français et en anglais mais la communication se trouvait fréquemment parasitée par des questions concernant la terminologie ou la traduction d'une terminologie très spécifique, même si le traducteur était habitué à travailler dans le domaine de la formation. En outre, il fallait distinguer 
les incompréhensions liées à la langue de celles liées à un concept. Ainsi, l'un des partenaires nous a posé à plusieurs reprises des questions sur le terme « pseudomot ${ }^{8} »$, que nous avons interprétées comme des questions linguistiques et auxquelles nous avons répondu par des exemples qui ont semblé le satisfaire... pour nous rendre compte, quelques mois plus tard, qu'il remettait en cause le principe même du recours aux pseudomots et pas seulement la terminologie, étant donné que l'écriture de sa langue est très phonographique.

Dans la mesure où l'objet de la recherche était l'illettrisme, les difficultés linguistiques étaient amplifiées par les questions d'écriture. Certes le système d'écriture des langues du projet, sauf le grec, a pour base l'alphabet latin, mais il est adapté ou enrichi, selon les langues, de digraphes et de diacritiques. D'autre part, la valeur phonologique d'une lettre ou d'un diacritique n'est pas identique d'une langue à l'autre, l'orthographe est plus ou moins phonographique selon les langues, les marques morphographiques plus ou moins régulières : chaque système linguistique comporte donc en lui-même les problèmes auxquels se heurteront les personnes en difficulté sur l'écrit. Grande est alors la tentation de renvoyer chaque partenaire à ses propres problèmes linguistiques et scripturaux.

Une seconde difficulté est vite apparue au cours des premiers échanges : la notion même d'illettrisme était loin de faire l'unanimité. Les définitions s'appuyaient sur des usages et des comportements sociaux, sur des traditions scolaires et culturelles, parfois aussi sur des normes institutionnelles ou des stéréotypes véhiculés par des articles grand public. Le terme même d'illettrisme n'avait pas d'équivalent en anglais, les partenaires utilisant soit « analphabetism » soit « illitteracy »; les représentants français et belges se référaient à la définition officielle de l'illettrisme proposée par l'ANLCI ${ }^{9}$ et publiée en 2003, mais le but n'était pas d'imposer la vision d'un pays ou d'un organisme. Très vite, après un état des lieux dirigé par le partenaire danois, le groupe de travail de MODEVAL1 a choisi la notion de « compétences de base » et non d' « illettrisme », prenant d'ailleurs en compte un texte de la

8. Pseudomots : mots qui n'existent pas dans la langue, mais dont la forme est plausible.

9. ANLCI (2003) : Lutter ensemble contre l'illettrisme. Cadre national de référence, p. 72. Disponible en ligne sur <http://www.anlci.gouv.fr/fileadmin/ Medias/PDF/EDITIONS/cadre_de_reference.pdf $>$. 
communauté européenne ${ }^{10}$ sans toutefois adopter le terme de «compétences clés » recommandé. Un degré qualitatif a été franchi durant MODEVAL2 à l'issue d'un long débat sur la notion de compétence au sein d'organismes européens et internationaux.

En 2005, l'état des lieux du partenaire danois ${ }^{11}$ est significatif des disparités sociales, culturelles et linguistiques au sujet de l'illettrisme dans les institutions enquêtées : parmi de nombreuses remarques, nous retiendrons que c'est le plus souvent la lecture (et non l'écriture) qui est testée, avec une attention spécifique à la compréhension, mais la vitesse de lecture est négligée, sauf au Danemark; il semble qu'une question de l'enquête concernant les compétences sociales et l'illettrisme ait été mal comprise, une partie des répondants ayant assimilé « compétences sociales » et «vie quotidienne », mais le rédacteur danois précise que ces questions sont considérées comme importantes par les partenaires pour la construction de l'outil d'évaluation; les partenaires maltais et hongrois indiquent qu'il n'existe pas chez eux d'évaluation spécifique pour les adultes; enfin les dispositifs d'évaluation des « adultes de bas niveau » sont extrêmement différents d'un pays à l'autre, voire d'une région à l'autre comme en Allemagne.

\section{Des échanges fructueux et des principes communs}

Les réunions, au cours desquelles nous avons pu échanger, ont permis aux participants de préciser ces résultats qui, encore une fois, soulignent la disparité des pratiques au sein d'un groupe somme toute assez réduit. Les chercheurs de l'université de Barcelone, par exemple, expérimentent en accompagnant les personnes illettrées durant leurs divers déplacements (à la poste, pendant les courses, au travail) pour observer et analyser les conduites de ces personnes in situ. Ceux de l'université de l'Égée utilisent des documents fonctionnels réels pour évaluer les niveaux de compréhension des personnes. Nous-mêmes, à l'université Lyon 2, fabriquons des documents proches de documents de la vie quotidienne, en tout cas nous privilégions les documents non scolaires. À l'Institut d'orthophonie du Jutland (Danemark), on préconise l'emploi

10. Commission européenne (2005). Proposition de recommandation du parlement européen et du conseil sur les compétences clés pour l'éducation et la formation tout au long de la vie, p. 2.

11. <http://www.modeval.org >. 
de tests orthophoniques normalisés ; à l'université de Kaposvar, en 2005, les partenaires hongroises évaluaient les tziganes adultes de la région avec des tests destinés aux jeunes enfants hongrois.

Après avoir pris acte de la diversité des pratiques, le PsyEF, qui, pour MODEVAL1, était chargé de la construction de l'outil d'évaluation, a estimé qu'il fallait valider un certain nombre de principes essentiels et élaborer un schéma général (voir ci-dessous) des compétences sur l'écrit avant de commencer à construire des épreuves. Un vote unanime lors de la deuxième réunion a d'ailleurs été exigé par le coordinateur afin de s'assurer de la participation volontaire de tous les partenaires.

I- Compréhension $\longrightarrow$ Compréhension de l'oral

II- Traitement de l'écrit $\left\{\begin{array}{l}\text { Production écrite } \begin{array}{l}\text { Assemblage (mots + pseudomots) } \\ \text { Adressage (mots + orthographe) }\end{array} \\ \text { Lecture } 2 \text { Compréhension (mots + textes simples) }\end{array}\right.$

III- Usage social de l'écrit

questionnaire

IV- Métacognitif $\longrightarrow$ questionnaire

Schéma général des compétences à évaluer

Si le premier principe (évaluation de la compréhension de l'oral) que nous souhaitions poser est largement acquis en 2011, il n'en allait pas de même six ans auparavant; notre expérience au niveau national nous permettait d'affirmer que l'on ne peut évaluer la compréhension de l'écrit sans s'assurer de la compréhension de l'oral. Or, en 2005, nombre de tests en littératie ne comprenaient pas d'épreuve d'oral. Les partenaires qui n'évaluaient pas l'oral habituellement ont assez rapidement accepté les arguments que nous avancions mais ils ont soulevé une question importante, à laquelle nous nous étions d'ailleurs heurtés lors de l'enquête $\mathrm{IVQ}^{12}$ : comment élaborer puis évaluer les critères de la

12. IVQ : enquête de l'Insee intitulée Information Vie Quotidienne, réalisée en 2004, qui concerne l'évaluation des compétences des adultes pour écrire, communiquer et compter. Elle a porté sur environ 10400 foyers. 
compréhension de l'oral lorsque l'on n'est pas linguiste? Nous avons rappelé que l'évaluation de la compréhension dans ce cadre n'a pas de prétention orthophonique; il s'agit simplement de s'assurer que ce n'est pas la méconnaissance de la langue qui est responsable d'une mauvaise performance à l'écrit. Les discussions ont alors porté sur le niveau exigible de maitrise de l'oral. L'un des partenaires avait participé à l'élaboration de PISA ${ }^{13} 2000$ et a proposé de s'en inspirer. Le groupe a trouvé plus opportun d'utiliser un outil européen, le cadre européen commun de référence pour les langues ${ }^{14}$. Pour déterminer un niveau plancher de maitrise de l'oral, en-deçà duquel les locuteurs ne pouvaient pas réussir les épreuves écrites que nous proposions, tous les partenaires du projet ont choisi le niveau B2 ${ }^{15}$. D'autres principes, comme la passation en face à face et non en collectif, comme la participation de la personne à son évaluation (co-évaluation dynamique ${ }^{16}$ ) ont été validés malgré des objections le plus souvent matérielles : coût des passations, locaux. L'écueil le plus grave était le manque de formation des formateurs à l'évaluation, dénoncé dans tous les organismes représentés.

Le projet MODEVAL2 devait combler ce manque. Cet outil de formation devait être adaptable aux différents publics visés par le projet (formateurs en illettrisme et alphabétisation, décideurs, responsables de centres de formation, chercheurs dans le domaine de l'évaluation des savoirs de base, statisticiens et personnes chargées d'enquêtes, etc.) et leur permettre d'élaborer eux-mêmes leurs propres outils d'évaluation à partir des recommandations du projet MODEVAL1. La construction du module de formation s'est effectuée en plusieurs temps et en concertation avec les partenaires européens : en premier lieu l'élaboration d'un cadre méthodologique dont était responsable le PsyEF (définition des

13. PISA : Programme for International Student Assessment : enquête de l'OCDE auprès des jeunes de 15 ans dans de nombreux pays (de 41 à 74 selon les années).

14. Cadre européen commun de référence pour les langues. Conseil de l'Europe. $<$ http://www.coe.int/t/dg4/linguistic/cadre_fr.asp $>$.

15. Peut comprendre le contenu essentiel de sujets concrets ou abstraits dans un texte complexe, y compris une discussion technique dans sa spécialité. Peut communiquer avec un degré de spontanéité et d'aisance tel qu'une conversation avec un locuteur natif ne comportant de tension ni pour l'un ni pour l'autre. Peut s'exprimer de façon claire et détaillée sur une grande gamme de sujets, émettre un avis sur un sujet d'actualité et exposer les avantages et les inconvénients de différentes possibilités.

16. Co-évaluation dynamique, voir J.-M. Besse, M.-H. Luis et al. (2004) : DMA, guide pratique, Paris, Retz. 
compétences en littératie dans un contexte européen), puis la production d'un matériel pédagogique pertinent faisant appel à la documentation propre à chaque pays (cadre théorique de l'outil MODEVALl pour évaluer les compétences de base en littératie), testing de la pertinence et la validité (supervisé par les partenaires espagnols).

Le projet CEPPAC3 avait pour objet de contribuer à améliorer les compétences des acteurs de formation. Il n'est pas souhaitable de construire une formation sans l'indication de sa durée, mais les partenaires, se fondant sur les habitudes de formation de leur pays, proposaient des durées soit trop courtes soit trop longues par rapport aux budgets généralement consentis. Nous avons alors travaillé en groupes pour envisager toutes les activités à proposer à des formateurs en formation. Nous sommes parvenus à une durée beaucoup plus importante que ne l'imaginaient la plupart des partenaires. La formation proposée dure de 6 à 8 jours en une, deux ou trois sessions. Un texte, appelé « document de référence » constitue une synthèse critique de CEPPAC2. Trois modules de formation proposent au formateur de formateurs des activités à réaliser avec ses formateurs-stagiaires. Un glossaire et une bibliographie complètent le document. De plus, dans chaque module, des renvois ont lieu vers un dossier d'annexes diverses et vers un document de ressources théoriques élaboré principalement par les partenaires espagnols et français. Pour ces ressources théoriques, le choix des auteurs et des textes proposés à la réflexion des formateurs a donné lieu à des discussions comparatives intéressantes. Les partenaires ont suivi la théorie linguistique de Nina Catach : tout système graphique est un plurisystème. Les travaux de Jack Goody, qui démontrent l'importance de l'écriture dans l'évolution de la pensée, sont précieux pour une formation non utilitariste à l'écrit. Ont été convoqués également des sociologues qui reconnaissent des compétences aux personnes en difficulté avec le langage et l'écriture comme Berstein, Labov et Enzensberger. Nous avons présenté aussi la pensée d'Emilia Ferreiro pour laquelle l'écriture est le produit d'une représentation et d'une construction par le sujet. Enfin des spécialistes de l'andragogie comme Paulo Freire et Malcom Knowles permettent aux formateurs de réfléchir aux aptitudes et aux dispositions du public en situation d'illettrisme. Pour compléter cet apport théorique chaque pays participant a fourni une bibliographie spécifique dans sa langue. Les documents édités sont disponibles en ligne ${ }^{17}$.

17. En ligne sur $<\mathrm{http}: / /$ www.ceppac.eu/>. 


\section{Pour une formation de formateurs interdisciplinaire}

Comme nous l'avons vu, les divergences au départ entre les partenaires concernaient aussi bien la définition de l'illettrisme que son évaluation ou sa remédiation. Mais, si des questions linguistiques, comme par exemple la spécificité du système orthographique, ne peuvent se réduire à une approche commune, de nombreux points de convergence sont apparus au cours des cinq années de collaboration. L'évolution de la réflexion des équipes impliquées dans les trois projets nous incite à préconiser une formation interdisciplinaire des formateurs des publics en difficulté sur l'écrit, incluant, outre l'approche du système graphique, les concepts et les activités issus de la psychologie, la psycholinguistique, la sociologie et l'andragogie.

\section{Une expérience positive à poursuivre?}

Les textes de MODEVAL et de CEPPAC sont en ligne et peuvent donc être consultés librement par les acteurs de la formation des adultes en difficulté. Toutefois ils sont protégés par des droits d'auteurs et ne peuvent être utilisés à des fins autres que personnelles sans l'autorisation du coordinateur des projets. C'est une garantie pour les auteurs qui pourraient craindre une mauvaise utilisation de l'outil et l'on sait qu'avec les personnes en difficulté les erreurs d'appréciation d'un formateur peuvent avoir de graves conséquences. Il faut bien reconnaitre, cependant, qu'il s'agit d'une limitation importante à l'utilisation de ces outils augmentée, semble-t-il, d'une certaine lenteur du chargement des documents engendrée peut-être par le niveau de protection. Signalons également que, depuis un ou deux ans, ce sont plutôt des formations en ligne interactives qui attirent les internautes bien qu'elles soient payantes la plupart du temps. Malgré tout, la gratuité de l'accès aux documents et l'originalité de la démarche restent des atouts non négligeables.

Notre participation active aux trois projets nous a permis de constater que les travaux du PsyEF étaient accueillis avec intérêt par nos partenaires européens et qu'en retour nous pouvions approfondir notre réflexion et améliorer notre matériel pédagogique grâce à de fructueux échanges. Compte tenu de l'évolution et de l'internationalisation des pratiques de formation, il nous parait nécessaire de maintenir des recherches au niveau européen, et à cet égard, les travaux des trois projets représentent une avancée certaine pour la réflexion sur les compétences des personnes en difficulté et sur les profils de formateurs. 


\section{RÉFÉRENCES BIBLIOGRAPHIQUES}

ANLCI (2003) : Lutter ensemble contre l'illettrisme. Cadre national de référence. Disponible en ligne sur <http://www.anlci.gouv.fr/fileadmin/ Medias/PDF/EDITIONS/cadre_de_reference.pdf $>$.

Besse J.-M., Luis M.-H., Paire K., Petiot-Poirson K. et Petit Charles E. (2004) : Évaluer les illettrismes. Diagnostic des modes d'appropriation de l'écrit : guide pratique, Retz, Paris.

Besse J.-M., Luis M.-H., Bouchut A.-L. et Martinez F. (2010) : « La mesure des compétences en traitement de l'écrit chez des adultes en grande difficulté », Économie et Statistique, $\mathrm{n}^{\text {os }}$ 424-425, p. 31-48. Disponible en ligne sur <http://www.insee.fr/fr/ffc/docs_ffc/ES424-425B. pdf $>$.

Besse J.-M., Luis M.-H. (2010) : « La conception du module d'orientation, du module de compréhension orale et du module ANLCI », encadré 4, Économie et Statistique, $\mathrm{n}^{\text {os }}$ 424-425, p. 18-19. Disponible en ligne sur <http://www.insee.fr/fr/ffc/docs_ffc/ES424-425A.pdf>.

\section{ANNEXE}

Le tableau ci-contre récapitule le partenariat européen autour de la formation d'adultes. Les organismes qui y sont répertoriés font ici l'objet d'une présentation détaillée :

(a) Thüringer Volkshochschulverband : Union des universités populaires de Thuringe.

(b) IFAPME : Institut wallon de formation en alternance des indépendants et petites et moyennes entreprises.

(c) HERB : Haute École Catholique du Luxembourg Blaise Pascal.

(d) Taleinstituttet Region Nordjylland : Institut linguistique de Nord Jutland (aphasies, dyslexies).

(e) Agora (Lorient) : entreprise d'économie sociale et familiale. Radar B (Lorient) : association pour la mise en œuvre de projets de développement culturels et éducatifs en Europe.

(f) ANLCI : Agence Nationale de Lutte Contre l'Illettrisme.

(g) PsyEF : Laboratoire Santé, Individu, Société (SIS, E.A.M. 4128), dirigé par le $\mathrm{P}^{\mathrm{r}}$ Yves Matillon (université de Lyon).

(h) MCAST : Malta College of Arts, Science and Technology.

(i) Espace femmes, Espace Mosaïque : centres de formation d'adultes à Fribourg et Lausanne. 


\begin{tabular}{|c|c|c|c|c|c|c|c|c|c|c|}
\hline 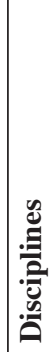 & 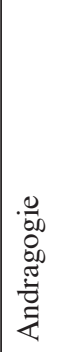 & 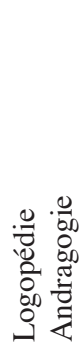 & $\begin{array}{l}\frac{0}{3} \\
\frac{0}{0} \\
\frac{0}{0} \\
\frac{0}{0}\end{array}$ & $\begin{array}{l}\frac{0}{00} \\
00 \\
\frac{0}{0} \\
0 \\
0 \\
0\end{array}$ & 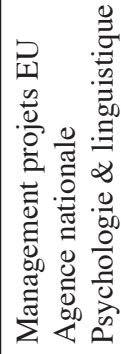 & 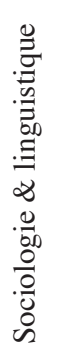 & $\begin{array}{l}00 \\
00 \\
00 \\
00 \\
00 \\
0 \\
0\end{array}$ & $\begin{array}{l}\frac{0}{00} \\
0 \\
\frac{0}{0} \\
\frac{0}{0} \\
0 \\
\tilde{n}\end{array}$ & $\begin{array}{l}\frac{0}{00} \\
\frac{0}{0} \\
\cdot \frac{0}{8} \\
0 \\
\mathscr{0}\end{array}$ & 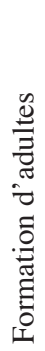 \\
\hline 范 & 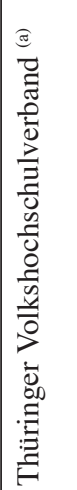 & 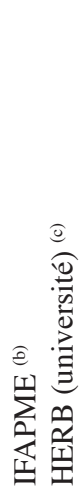 & 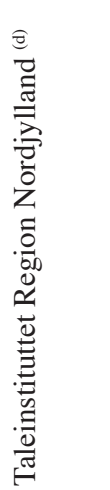 & 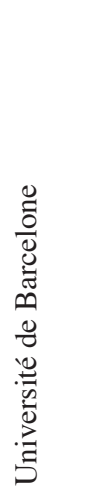 & 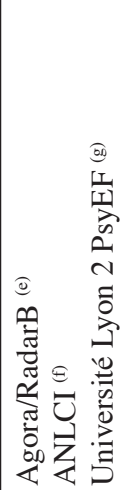 & 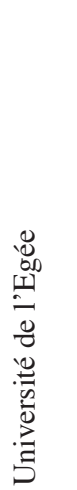 & 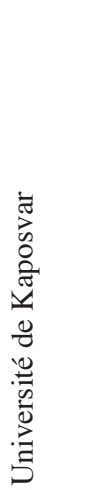 & 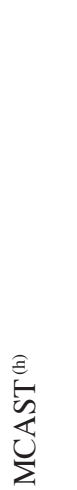 & 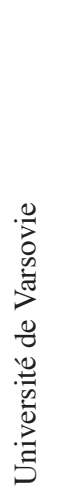 & 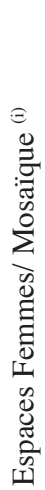 \\
\hline 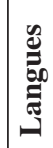 & 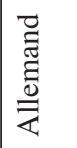 & 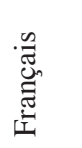 & $\begin{array}{l}\frac{a}{0} \\
\stackrel{\Xi}{0} \\
0\end{array}$ & 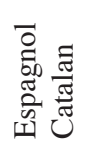 & 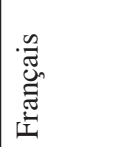 & $\begin{array}{l}\text { U. } \\
\dot{0}\end{array}$ & 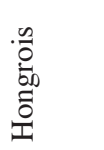 & 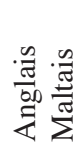 & $\begin{array}{l}.0 \\
0 \\
0 \\
0 \\
0\end{array}$ & 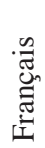 \\
\hline 赹 & 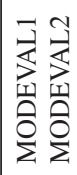 & 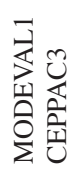 & 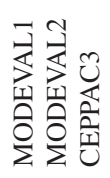 & 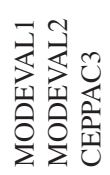 & 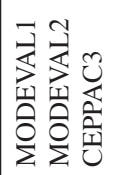 & 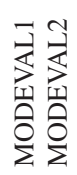 & 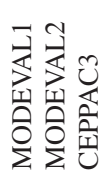 & 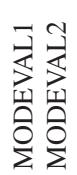 & 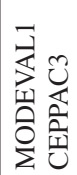 & 究 \\
\hline$\sum_{0}^{\infty}$ & 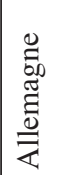 & $\begin{array}{l}\mathscr{0} \\
\frac{\tilde{\sigma}}{00} \\
\frac{0}{0}\end{array}$ & 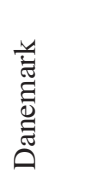 & 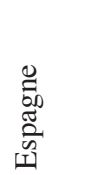 & 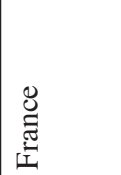 & 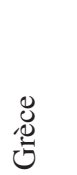 & 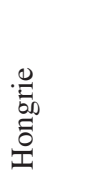 & $\frac{\stackrel{\Perp}{\pi}}{\stackrel{\pi}{\Sigma}}$ & $\begin{array}{l}0 \\
\tilde{0} \\
0 \\
0 \\
0\end{array}$ & $\begin{array}{l}\mathscr{D} \\
\ddot{\Xi} \\
\tilde{\omega}\end{array}$ \\
\hline
\end{tabular}

Tableau récapitulatif du partenariat 
